\title{
Rabaska
}

Revue d'ethnologie de l'Amérique française

\section{SAVARD, Félix-Antoine. Menaud Maître-draveur. Édition critique par Yvan G. LEPAGE. Montréal, Presses de l'Université de Montréal, « Bibliothèque du Nouveau-Monde ", 2004, 782 p. ISBN 2-7606-1884-6}

\section{André Gaulin}

Volume 4, 2006

URI : https://id.erudit.org/iderudit/201794ar

DOI : https://doi.org/10.7202/201794ar

Aller au sommaire du numéro

Éditeur(s)

Société québécoise d'ethnologie

ISSN

1703-7433 (imprimé)

1916-7350 (numérique)

Découvrir la revue

Citer ce compte rendu

Gaulin, A. (2006). Compte rendu de [SAVARD, Félix-Antoine. Menaud Maitre-draveur. Édition critique par Yvan G. LEPAGE. Montréal, Presses de l'Université de Montréal, « Bibliothèque du Nouveau-Monde », 2004, 782 p. ISBN 2-7606-1884-6]. Rabaska, 4, 191-193. https://doi.org/10.7202/201794ar d'utilisation que vous pouvez consulter en ligne. 
SAVARD, Félix-Antoine. Menaud Maître-draveur. Édition critique par Yvan G. LePage. Montréal, Presses de l'Université de Montréal, « Bibliothèque du Nouveau-Monde $», 2004,782$ p. ISBN 2-7606-1884-6.

Quand paraît l'édition critique du roman célèbre de Félix-Antoine Savard à la toute fin de 2004, elle s'ajoute à la quarantaine déjà parue dans la riche "Bibliothèque du Nouveau Monde " où figurent des titres de toutes les périodes allant de Jacques Cartier et Étienne Parent à Alain Grandbois et Jacques Ferron. Il faut savoir gré au professeur Yvan Lepage d'avoir eu le courage de s'attaquer à cette entreprise ardue d'établir l'édition critique du roman lyrique que Savard fit paraitre en 1937 et qu'il retoucha constamment pendant trente ans, jusqu'à sa version de 1967 qu'il voulut définitive. Savard avait alors 71 ans. On croit facilement Lepage qui écrit d'entrée de jeu de 
son chapitre « Établissement du texte » que « Peu d'œuvres ont une histoire génétique et textologique aussi riche et aussi complexe que Menaud MaîtreDraveur ».

En effet, Lepage distingue huit éditions du roman savardien, la première étant l'édition princeps de 1937. Une deuxième version, celle de 1938, supprime les mots du lexique québécois en italique du Menaud pour les regrouper dans un "Petit glossaire" final. L'Action catholique publie en 1939 une troisième édition constituée de vingt-cinq feuilletons de quatre pages. La quatrième version de 1944, dite "définitive ", comporte des changements importants non pas au niveau spatio-temporel et des personnages mais pour l'épuration des images, ce que Lepage appelle une " édition édulcorée », et curieusement longtemps disponible par les tirages de 1945, 1948,1949 et 1953. La cinquième édition de 1957, pour la collection du "Nénuphar ", ajoute une quinzaine de corrections et de modifications à la précédente. Avec la sixième édition de 1960, Savard dit revenir à l'édition originale, ce qui pour Lepage n'est pas tout à fait le cas même si cette version s'en inspire largement. En 1964, Savard produit une septième version pour la collection " Alouette bleue », issue de la copie annotée de la sixième par des corrections au feutre noir ainsi qu'au stylo à bille bleu ou noir. Savard y a inscrit sur la couverture d'une chemise : « Menaud / Édition / dernière / définitive, celle-là, été 1964 » Il y aura pourtant une huitième édition, celle de 1967, dont Lepage fera "le texte de base de son édition critique ", respectant ainsi la volonté de Félix-Antoine Savard qui voyait son œuvre de 1937 ainsi couronnée comme une réalisation formelle plutôt qu'idéologique.

Ce texte central de l'édition critique de Menaud Maître-Draveur est d'autant plus précieux qu'il n'a paru jusqu'à ce jour qu'avec l'édition de luxe de 1967. Il nous est donc livré ici prioritairement comme version finale, fruit des nombreuses refontes qu'en fit Savard après huit versions et plusieurs " états » (parfois lacunaires, car Savard a éparpillé parfois ses manuscrits, en faisant don à des amis) du texte du roman, Lepage en ayant distingué douze. Outre les huit versions citées plus haut, il faut ajouter le manuscrit A et le manuscrit B du roman de 1937, des prépublications de chapitres dans le journal du séminaire de Chicoutimi, L'Alma mater, un manuscrit $\mathrm{C}$ de l'édition princeps ainsi que des jeux préparatoires des $4^{c}$ et $7^{\mathrm{e}}$ éditions. On en admirera d'autant le travail patient - devenant parfois inextricable dans son interprétation - qu'il faut y ajouter encore des variantes entre le jeu préparatoire ou les manuscrits et les éditions ! C'est pourquoi Lepage opte pour une édition génétique plutôt que diplomatique parce que « La critique génétique nous a appris à respecter le caractère propre des avant-textes ", écrit-il. Alors que l'édition critique diplomatique s'en remet rigoureusement au texte, simple reproduction, la critique génétique en fait une « transcription 
dynamique ", l'éditeur critique faisant ainsi son travail plutôt que de s'en remettre au lecteur qui n'aura souvent pu faire le chassé-croisé des textes et de leur évolution.

D'ailleurs, le professeur Yvan Lepage a jugé qu'au moins cinq versions des douze états de Menaud Maître-Draveur étaient « irréductibles », soit les deux premières versions manuscrites, les éditions de 1937 (princeps), de 1944

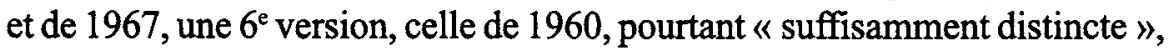
ayant été prise en compte en variante de l'édition princeps. Ces états étaient incompressibles et permettaient à l'éditeur critique « de rendre compte au plus près du travail d'écriture savardien, tout en rendant sensibles les tensions du texte entre sa clôture définitive et ses diverses métamorphoses ». Le lecteur de ce monumental ouvrage trouvera donc ici l'édition critique du texte de 1967 rendue ainsi disponible et, dans l'esprit de Savard, la copie enfin rendue stylistiquement à terme de son Menaud. Le lecteur trouvera quatre versions en annexe : éditions génético-critiques des manuscrits $A$ et $B$, éditions diplomatiques de 1937 et 1957, tous états irréductibles associés à des versions voisines dans ces douze états du texte évoqués plus haut.

Voilà le travail colossal accompli par Yvan Lepage qui nous permettra de relire Menaud Maître-Draveur dans son évolution stylistique, puisque Savard voyait d'abord son œuvre comme littéraire même si les interprètes des idéologies y trouveront leur profit puisque, quoi qu'il en dise, monseigneur Savard ne fut pas insensible à la critique qu'on fit diversement de son Menaud d'une décennie à l'autre, tourmenté qu'il fut aussi par ceux qui le forcèrent, bien malgré lui, à écrire un testament politique. Là-dessus, le lecteur de ce nouveau joyau de la « Bibliothèque du Nouveau Monde » trouvera aussi dans ce livre une longue «Introduction » d'une cinquantaine de pages qui situe bien Félix-Antoine Savard, homme et écrivain, en son temps. On y apprend beaucoup des choses du fils de famille, du jeune prêtre d'abord un peu récalcitrant et entré un moment chez les Bénédictins, du colonisateur en Abitibi, de l'homme des bois qui marchait Charlevoix aux confins du comté de Chicoutimi, du conservateur croyant qui avait sa vision du Canada français, des autres œuvres écrites de l'auteur où le roman de 1937 est souvent évoqué, surtout dans ses écrits plus autobiographiques. On ne s'étonnera d'ailleurs pas que Lepage soit à préparer une biographie du grand prosateur et poète québécois inhumé dans son cher Charlevoix, tellement sa vision de Savard sait faire la part de l'homme, de l'écrivain, de sa réception en fonction de notre évolution dans l'Histoire. Une bibliographie remarquable termine ce livre savant de près de 800 pages qui fait enfin le point sur Menaud MaîtreDraveur. 